\title{
Desarrollo profesional de profesores de matemáticas en ambientes virtuales: ventajas, aproximaciones teóricas y futuras líneas de investigación
}

Online mathematics teachers' professional development: advantages, theoretical perspectives and future investigation lines

\section{Fátima Widman Aguayo ${ }^{1}$}

Resumen: Los ambientes virtuales han demostrado ser un espacio en donde el desarrollo profesional de docentes de matemáticas puede llevarse a cabo. Esta modalidad formativa ha cobrado importancia debido al rápido crecimiento en su oferta y demanda. Sin embargo, aún se sabe poco sobre cómo se aprovechan estos espacios en línea para favorecer el desarrollo profesional de los profesores de matemáticas. En este ensayo se presenta una revisión de literatura que sintetiza qué avances se han tenido en la investigación de esta área en la última década. Particularmente, se identifican cuáles son los principales argumentos por los que se piensa que los escenarios en línea son adecuados para promover el desarrollo profesional de los docentes de matemáticas; qué referentes teóricos se han utilizado para el diseño y análisis de las actividades formativas que promueven este tipo de desarrollo profesional, y cuáles son aquellos aspectos relevantes que no han sido suficientemente investigados con relación a este tema.

Palabras clave: desarrollo profesional de docentes de matemáticas, formación docente en línea, educación a distancia en línea, profesores en servicio, tecnologías digitales.

Fecha de recepción: 03 de julio de 2017. Fecha de aceptación: 08 de junio de 2021.

1 Instituto Politécnico Nacional, CICATA Legaria, Programa de Matemática Educativa / Centro de Estudios Tecnológicos Industrial y de Servicios 112, fatimawidman@hotmail.com, orcid.org/0000-0003-4040-8900 


\begin{abstract}
Virtual environments have proven to be a space where the professional development of mathematics teachers can take place. This training modality has gained importance due to the rapid growth in its supply and demand. However, little is yet known about how these online spaces are used to promote the professional development of mathematics teachers. This essay presents a literature review that synthesizes what advances have been made in this research area during the last decade. In particular, I identify the main arguments why online settings are thought to be adequate to promote the professional development of mathematics teachers; what theoretical references have been used for the design and analysis of the training activities that promote this type of professional development, and what are the relevant aspects that have not been sufficiently investigated in relation to this topic.
\end{abstract}

Keywords: online mathematics teacher's education, professional development, in service mathematics teachers, digital technologies, professional learning experiences.

\title{
INTRODUCCIÓN
}

El estudio del desarrollo profesional de los docentes de matemáticas se ha consolidado como un área de investigación dentro de la matemática educativa. Esto se hace evidente al encontrar en la literatura científica un extenso número de publicaciones que abordan diferentes problemáticas o aspectos relativos a este tema. Particularmente, se ha notado un creciente interés en el estudio del desarrollo profesional de profesores de matemáticas dentro de escenarios virtuales o en línea. Lo anterior, debido al rápido aumento en la oferta y demanda de programas formativos en línea dirigidos a estos docentes (Gueudet et al., 2012). Sin embargo, aún se sabe poco sobre cómo se aprovechan estos espacios en línea para favorecer el desarrollo profesional de dichos profesores (Cendros y Gadanidis, 2020). Por lo que, en este artículo se presenta una revisión de literatura que permita sintetizar qué avances se han tenido en la investigación de esta área en la última década y qué aspectos aún quedan pendientes por investigar.

Específicamente, la revisión de literatura presentada, se enfoca en: (1) identificar cuáles son los principales argumentos por los que se piensa que los 
escenarios en línea son adecuados para promover el desarrollo profesional de los docentes de matemáticas; (2) cuáles son los referentes teóricos que se han utilizado para el diseño y análisis de las actividades formativas que promueven este tipo de desarrollo profesional, y finalmente (3) cuáles son aquellos aspectos relevantes que no han sido suficientemente investigados con relación a este tema.

\section{MÉTODO}

En esta sección se presenta el método que se siguió para desarrollar la revisión de literatura que se presenta en este escrito. Particularmente, se describen qué tipos de fuentes de información se consultaron y bajo qué criterios fueron seleccionadas.

\section{FUENTES DE INFORMACIÓN}

Para la revisión bibliográfica realizada se consideraron tres tipos de fuentes de información: revistas especializadas, memorias de congresos de matemática educativa y libros relativos al desarrollo profesional docente de docentes de matemáticas.

En cuanto a las revistas especializadas, se seleccionaron aquellas con mayor prestigio dentro del campo de la matemática educativa con la intención de procurar la calidad y confiabilidad de la información obtenida. Para identificarlas, se utilizaron tres rankings de revistas de investigación que se han generado en esta disciplina: Toerner y Arzarello (2012), Williams y Leatham (2017), y Andrade-Molina et al., (2020). En la tabla 1, se presentan los nombres de las revistas incluidas en los rankings en donde se encontró al menos un artículo relativo al desarrollo profesional de docentes de matemáticas en línea. 
Tabla 1. Número de artículos relativos al desarrollo profesional docente en línea encontrados en las revistas seleccionadas.

\begin{tabular}{l|c}
\hline \multicolumn{1}{c|}{ Revista } & $\begin{array}{c}\text { Número de artículos } \\
\text { identificados }\end{array}$ \\
\hline ZDM Mathematics Education & 10 \\
\hline Educational Studies in Mathematics & 3 \\
\hline $\begin{array}{l}\text { Revista Latinoamericana de Investigación en } \\
\text { Matemática Educativa (RELIME) }\end{array}$ & 2 \\
\hline Boletim de Educaçāo Matemática (BOLEMA) & 1 \\
\hline Journal of Mathematics Teacher Education & 1 \\
\hline Mathematics Education Research Journal & 1 \\
\hline Research in Mathematics Education & 1 \\
\hline School Science and Mathematics (SSM) & 1 \\
\hline UNIÓN. Revista Iberoamericana de Educación & 1 \\
Matemática & \\
\hline
\end{tabular}

De igual forma, se revisaron las memorias de los grupos de trabajo relativos al desarrollo profesional docente en línea en los congresos: ICME (International Congress on Mathematical Education), PME-NA (Psychology of Mathematics Education - North America), CERME (Congress of European Research in Mathematics Education), RELME (Reunión Latinoamericana de Matemática Educativa) y CIAEM (Conferencia Interamericana de Educación Matemática). Dichos congresos fueron seleccionados debido a que sus memorias se encuentran accesibles para ser consultadas. En la tabla 2, se presentan los congresos en donde se encontró al menos un artículo relativo al desarrollo profesional de docentes de matemáticas en línea. 
Tabla 2. Número de artículos identificados en las memorias de los congresos seleccionados.

\begin{tabular}{l|c|l|c|l|c}
\hline \multicolumn{1}{c|}{ Congreso } & Artículos & Congreso & Artículos & Congreso & Artículos \\
\hline ICME 13 & 1 & RELME 26 & 1 & PME-NA 34 & 2 \\
\hline CERME 11 & 2 & RELME 28 & 2 & PME-NA 36 & 1 \\
\hline CERME 9 & 1 & RELME 31 & 2 & PME-NA 37 & 1 \\
\hline CERME 7 & 1 & XIV CIAEM & 2 & PME-NA 38 & 2 \\
\hline CERME 6 & 1 & PME-NA 31 & 3 & PME-NA 39 & 1 \\
\hline RELME 22 & 1 & PME-NA 32 & 1 & PME-NA 40 & 1 \\
\hline RELME 24 & 2 & PME-NA 33 & 1 & PME-NA 41 & 3 \\
\hline
\end{tabular}

Finalmente, se consultaron varios libros especializados en desarrollo profesional docente, pero se seleccionaron únicamente aquellos que incluyeran al menos un capítulo relativo al desarrollo profesional de docentes en matemáticas en línea. En la tabla 3, se muestra tanto el nombre de estos libros como el nombre de los capítulos seleccionados dentro de ellos.

Tabla 3. Nombre de libros y capítulos seleccionados relativos al desarrollo profesional docente en línea.

\begin{tabular}{l|l}
\multicolumn{1}{c}{ Libro } & \multicolumn{1}{c}{ Capítulos identificados } \\
\hline $\begin{array}{l}\text { The Professional Education and } \\
\text { Development of Teachers of Mathematics }\end{array}$ & $\begin{array}{l}\text { Tools and Settings Supporting Mathematics } \\
\text { Teachers' Learning in and from Practice }\end{array}$ \\
\hline Learning Through Teaching Mathematics & $\begin{array}{l}\text { Dialogical Education and Learning } \\
\text { Mathematics Online from Teachers }\end{array}$ \\
\hline
\end{tabular}

\section{CRITERIOS DE SELECCIÓN}

Para realizar la búsqueda en las fuentes previamente mencionadas se utilizaron las siguientes palabras clave: online professional development, web based professional development, online mathematics teacher education, internet professional development, formación docente en línea y formación continua de profesores a distancia. De los artículos resultantes en la búsqueda por palabras 
claves, se seleccionaron aquellos que cumplieran los siguientes tres criterios: (1) que el artículo esté escrito en idioma inglés, español o portugués, (2) que la temática abordada sea relativa al desarrollo profesional de docentes de matemáticas dentro de ambientes virtuales, y (3) que las publicaciones se encuentren en el rango del 2009 al 2020. Se parte del 2009, por ser el año donde comienzan a reportarse estudios en congresos internacionales relativos a esta temática (Ponte et al., 2009) y se revisa hasta el 2020 con la intención de presentar información lo más actualizada posible.

Se considera importante aclarar, que dentro del artículo se podrán encontrar algunas referencias que no corresponden a las fuentes de información ni criterios de selección mencionados con anterioridad, pero que se incluyeron debido a que conformaron fuentes de consulta imprescindibles, ya que ampliaban la explicación de ciertas aproximaciones teóricas mencionadas en los artículos inicialmente revisados.

\section{CONCEPTUALIZACIÓN Y CLASIFICACIÓN DE PROCESOS DE DESARROLLO PROFESIONAL EN LÍNEA PARA DOCENTES DE MATEMÁTICAS}

Entre las actividades profesionales que un docente realiza, se encuentran aquellas destinadas a procurar su formación continua. Cuando estas actividades se llevan a cabo a través de entornos virtuales suelen nombrarse de diversas formas en la literatura científica, por ejemplo: formación continua a distancia, formación docente en línea, educación virtual para profesores, entre otras. En este artículo, a este tipo de actividades se les denominará actividades para el desarrollo profesional en línea de docentes de matemáticas y se conceptualizan, a partir de las propuestas de Kynigos y Kalogeria (2012) y Lezama (2009), como todo proceso formativo que se desarrolla en espacios virtuales y que permite al profesor cruzar los límites de sus comunidades académicas habituales y favorecer la problematización y mejora de su práctica a partir de la generación de espacios de aprendizaje, reflexión y diálogo y el trabajo con diferentes herramientas teóricas, metodológicas y tecnológicas.

El desarrollo profesional en línea de docentes de matemáticas puede clasificarse como formal o informal. Se le considera informal cuando se desarrolla a través de redes sociales (Facebook, Twitter, blogs, etc.) en donde los profesores interactúan y realizan acciones como compartir lecciones, sugerir y revisar recursos y participar en foros de discusión. Esto sin que exista un diseño instruccional 
de por medio, ni se espere la entrega específica de alguna evidencia de aprendizaje por parte de los profesores. Un ejemplo de este tipo de formación profesional se muestra en Bommel y Liljekvist (2015).

Si bien, en la literatura se hace explícito cuándo un proceso de desarrollo profesional docente se considera informal, no se menciona de forma evidente cuándo se considera formal. Sin embargo, a partir de lo mencionado anteriormente, podrían considerarse procesos formales de desarrollo profesional aquellos que cuentan con un diseño instruccional basado en modelos o teorías pedagógicas, y que solicitan a los profesores participantes evidencias de sus aprendizajes o procesos reflexivos a partir de su participación en foros, entregas de productos, elaboración de propuestas pedagógicas, etc.

\section{ARGUMENTOS POR LOS QUE SE PIENSA QUE LOS ESCENARIOS EN LÍNEA SON ADECUADOS PARA PROMOVER EL DESARROLLO PROFESIONAL DE DOCENTES DE MATEMÁTICAS}

Existen argumentos que permiten considerar al desarrollo profesional de docentes de matemáticas en escenarios virtuales como una buena práctica formativa incluso superior a la modalidad presencial (Surrette y Johnson, 2015) ya que posee características particulares que favorecen la formación de los docentes y que no pueden observarse en entornos presenciales (Dean y Silverman, 2009). Estos argumentos frecuentemente son asumidos y no siempre se mencionan de forma explícita (Li y Qi, 2011), por lo que a continuación, se presentan todos aquellos encontrados en la revisión realizada clasificados en tres categorías: institucionales, pedagógicos y relativas a la mejora profesional.

\section{ARGUMENTOS INSTITUCIONALES}

Se consideran argumentos institucionales a aquellos que presentan al desarroIlo profesional en línea como una alternativa para solucionar problemáticas institucionales y no se enfocan en aspectos específicos de la formación docente. Uno de estos argumentos es la facilidad de implementar este tipo de cursos a gran escala a través de la reproducción repetitiva de un mismo diseño instruccional. Esta opción ha resultado ser conveniente para dar a conocer a todos los docentes de un país tanto las modificaciones realizadas en los currículos nacionales como 
la introducción de nuevas estrategias pedagógicas, generalmente aquellas relacionadas con el uso de las nuevas tecnologías de la información y la comunicación (Maass et al., 2019).

Otro argumento institucional, es aquel que presenta al desarrollo profesional en línea como una alternativa que permite a aquellos profesores que laboran en áreas marginadas o distritos rurales, acceder fácilmente a las mismas opciones formativas que sus colegas ubicados en distritos más poblados, sin que esto represente una derrama excesiva de recursos económicos invertidos en transporte (Dean y Silverman, 2009; Lee et al., 2017).

Un último argumento institucional es que este tipo de formación continua posibilita que la participación de los profesores no se vea impedida por la variación en su disponibilidad de horario (Surrette y Johnson, 2015). Esta problemática es frecuente en algunos países porque la zona horaria cambia entre sus diferentes regiones, y en otros porque la carga laboral de los profesores suele ser excesiva o repartida entre varias escuelas, lo que dificulta su disponibilidad para reunirse a una determinada hora (Dean y Silverman, 2009; Montiel, 2010; Kynigos y Kalogeria, 2012). Si las actividades destinadas a la formación de los profesores son solicitadas de forma obligatoria por las instituciones educativas, la flexibilidad temporal que ofrecen los entornos virtuales representa una posibilidad para que un mayor número de profesores pueda participar en ellas, y cumplir de esta forma los requerimientos de su institución.

\section{ARGUMENTOS PEDAGÓGICOS}

Se consideran argumentos pedagógicos a aquellos que resaltan características de los ambientes virtuales que favorecen el aprendizaje de los profesores de matemáticas. Por ejemplo, Borba y Llinares (2012), y Clay et al. (2012) consideran que la comunicación escrita y asíncrona que caracteriza a las participaciones dentro de los foros virtuales provoca una mayor profundidad y riqueza en las reflexiones de los profesores, ya que permite que puedan leer y analizar con detenimiento las aportaciones previas de sus colegas y formular a partir de estas sus propias opiniones. Incluso, el dinamismo asíncrono de los foros facilita que los participantes consulten fuentes externas de información que les permitan clarificar y fundamentar de mejor forma sus aportaciones (Sánchez, 2010). De igual forma, se considera que es más fácil para los profesores realizar cuestionamientos a través de foros, ya que no necesariamente tienen que dirigirlos 
hacia una persona en particular (Erixon, 2016). Cada una de las acciones mencionadas (lectura reflexiva, redacción escrita, búsqueda de información, cuestionamientos, etc.), son factores determinantes en el aprendizaje de los profesores y se favorecen al participar en actividades propias de entornos virtuales.

Otro de los argumentos pedagógicos mencionados en la literatura es que la variabilidad y disponibilidad de recursos de apoyo que son proporcionados en este tipo de espacios formativos favorecen el logro de los aprendizajes. Por ejemplo, las características de los entornos virtuales permiten que dichos recursos permanezcan alojados de forma permanente en el aula virtual y de esta forma, es posible que el docente consulte los recursos deseados las veces que considere, procurando de esta forma una continua reestructuración y recontextualización de los contenidos (Müller et al.,2011).

Asimismo, los entornos virtuales permiten el acceso a materiales especializados que pueden llegar a ser difíciles de conseguir para los profesores y que muchas veces contienen la información más actualizada y relevante del área de interés (Kynigos y Kalogeria, 2012; Surrette y Johnson, 2015). Finalmente, los entornos virtuales permiten que un mismo contenido se presente a través de diversos formatos (videos, audios, textos, etc.), favoreciendo los diferentes estilos de aprendizaje que pudieran tener los participantes y enriqueciendo los canales de atención (Borba y Llinares, 2012; Patahuddin, 2013).

Finalmente, otro de los argumentos pedagógicos encontrados en la literatura, hace referencia a la capacidad de los entornos virtuales para transformar la manera en la que los profesores aprenden matemáticas (Clay et al., 2012). Por ejemplo, una determinada herramienta tecnológica puede permitir al profesor replantear o profundizar en la comprensión de cierto concepto matemático a partir del manejo de gráficas y de simuladores (Gueudet et al., 2012). Asimismo, dichas herramientas permiten que el profesor genere propuestas innovadoras para la presentación de un tema particular y a la vez, pueda cuestionar y discutir sobre sus propuestas y las de sus colegas, a través de espacios síncronos y asíncronos generando así procesos de aprendizaje colaborativos mediados por la tecnología. 


\section{ARGUMENTOS RELATIVOS A LA MEJORA PROFESIONAL}

Son aquellos que explican de qué forma la participación de los docentes en actividades de desarrollo profesional en línea impacta en su práctica profesional. Uno de ellos, afirma que este tipo de actividades formativas tienen un impacto positivo en las creencias de los docentes sobre el uso de la tecnología, por lo que es más probable que se vean motivados a incluirla dentro de su práctica (Patahuddin, 2013). Asimismo, Müller et al. (2011) afirman que estos espacios formativos permiten al docente familiarizarse con su funcionamiento, lo cual puede llegar a resultarles útil en caso de que se presentara la oportunidad o necesidad de integrar estas tecnologías a su práctica habitual.

Otro de los argumentos hace referencia al diseño instruccional de algunos de estos cursos, ya que generan alternativas de rutas o caminos profesionales y permiten que el profesor seleccione las actividades y temas con los cuales trabajar (Bommel y Liljekvist, 2015). Dicha flexibilidad genera una percepción de auto eficacia e independencia en los profesores (Patahuddin, 2013) y a la par, promueve la independencia y construcción de un camino profesional propio.

Finalmente, uno de los argumentos más mencionados en la literatura es la oportunidad que brindan las actividades de desarrollo profesional en línea para generar espacios de colaboración nacionales e internacionales entre docentes con intereses en común e incluso, con otros profesionales de la enseñanza (Surrette y Johnson, 2015; Gueudet et al., 2012; Li y Qi, 2011). Dicho intercambio cultural y profesional permite la construcción de comunidades virtuales en donde se generan espacios para compartir experiencias profesionales, desarrollar nuevas ideas curriculares y colaborar en proyectos pedagógicos. Asimismo, este tipo de comunidades propician que el desarrollo profesional de los profesores progrese de forma continua, lo que aumenta las probabilidades de que lo aprendido realmente impacte de forma positiva en su desempeño profesional (Clark-Wilson y Hoyles, 2019).

En conclusión, se ha argumentado sobre una variedad de factores que permiten asegurar que el desarrollo profesional en línea de docentes de matemáticas constituye una opción formativa no solo favorable sino deseable para los profesores. Sin embargo, se considera importante aclarar que todos los argumentos mencionados parten del supuesto de que las actividades de desarrollo profesional en línea en las que un docente participa son propuestas por un equipo de profesionales, a partir de diseños instruccionales que consideran los 
fundamentos teóricos, metodológicos y tecnológicos necesarios para un buen diseño e implementación.

\section{APROXIMACIONES TEÓRICAS IMPLEMENTADAS EN EL DESARROLLO PROFESIONAL DOCENTE EN LÍNEA}

Para que el desarrollo profesional pueda llevarse a cabo dentro de espacios virtuales requiere de un diseño instruccional en donde se determinen el o los propósitos formativos, las estrategias de aprendizaje, y los recursos didácticos necesarios. Adicionalmente, debido a su naturaleza virtual, se requiere disponer de diversas herramientas tecnológicas que permitan la participación e interacción de los participantes. En la literatura especializada se puede dar cuenta de cómo las perspectivas teóricas que se adoptan orientan la toma de ciertas decisiones en el diseño instruccional y a la par, sirven como lentes para el análisis de lo que ocurre en estos procesos formativos una vez que se han implementado, favoreciendo de esta forma la evaluación y mejora continua de dichos procesos.

En esta sección, se describen cuáles son las principales aproximaciones teóricas que se han empleado en los escenarios de instrucción virtual para el desarrollo profesional docente, clasificadas de acuerdo con la intención formativa para la que son utilizadas. Cabe aclarar que si bien, se mencionarán por separado, es posible encontrar más de una aproximación teórica en un mismo diseño instruccional, es decir, no son teorías necesariamente excluyentes entre sí.

\section{PROPÓSITOS FORMATIVOS}

El conocimiento de los contenidos matemáticos y la pedagogía para abordar dichos contenidos en las aulas son aspectos formativos de interés dentro del desarrollo profesional en línea de docentes de matemáticas. Por ejemplo, Clay et al. (2012) y Silverman y Clay (2009a) utilizan ciertas propuestas pedagógicas de Shulman (1986) para plantear los objetivos de aprendizaje dentro de su propuesta de formación virtual. Asimismo, en los últimos años, han aumentado aquellos propósitos formativos relativos a que el profesor de matemáticas incorpore el uso de la tecnología dentro de sus estrategias de enseñanza en el aula (Hoyos, 2012). Un ejemplo de lo anterior es el que presentan Clark-Wilson y 
Hoyles (2019), quienes adoptan el modelo PTK (Pedagogical Technology Knowledge) de Thomas y Hong (2013) en su propuesta formativa.

\section{INTERACCIÓN ENTRE MEDIOS}

En algunas propuestas formativas como la que presenta Borba (2012) se enfatiza que, si se busca generar conocimientos y colaboración dentro de ambientes virtuales, debe considerarse cómo los diferentes medios tecnológicos interaccionan con los humanos e impactan en sus producciones matemáticas. Es el caso del constructo teórico humans-with-media el cual asume que la tecnología puede afectar el tipo de conocimientos matemáticos producidos dentro de un ambiente de aprendizaje. Es decir, dicho constructo enfatiza que las tecnologías juegan un rol activo dentro del aprendizaje y se genera entre estas y los humanos una interrelación en donde cada parte resulta implicada (Borba y Llinares, 2012).

Otra aproximación teórica que considera el impacto de los medios en la formación profesional es la documentational approach propuesta por Gueudet y Trouche (2009). Bajo esta aproximación se considera que un proceso de génesis documental ocurre cuando los profesores utilizan diversos recursos y los transforman en nuevos ajustados a su práctica. Gueudet y Trouche (2009) afirman que el proceso de documentación es la parte central de cualquier actividad profesional de un docente, por lo que es importante enfocar la atención en cómo lo lleva a cabo a través de los recursos dispuestos en una experiencia de formación. Gueudet et al. (2012) consideran esta perspectiva teórica para observar la interacción entre los profesores y los recursos dentro de distintos entornos virtuales destinados al desarrollo profesional de docentes de matemáticas.

\section{FORMACIÓN DE COMUNIDADES}

Algunas de las propuestas formativas en línea destinadas al desarrollo profesional de profesores de matemáticas plantean, como una de sus principales intenciones diseñar espacios de encuentro entre grupos de profesores de matemáticas y ciertas comunidades de práctica (COP) constituidas por educadores matemáticos o bien generar nuevas comunidades de práctica entre los profesores participantes en un proceso formativo. Las COP conforman una teoría social de aprendizaje en donde se describen tres dimensiones mediante las 
cuales la práctica docente puede generar una comunidad: un compromiso mutuo, una empresa conjunta y un repertorio compartido (Wenger, 2001). Lee et al. (2017) mencionan que al conformar dichas comunidades los participantes extienden sus accesos a diferentes recursos y prácticas pedagógicas más allá de las que pueden encontrar en sus centros laborales y de igual forma, se favorecen acciones formativas permanentes entre ellos.

Una de las estrategias utilizadas para la generación de este tipo de comunidades es proponer el uso de distintos artefactos (tareas, recursos, proyectos, etc.) diseñados particularmente para servir como objetos frontera que faciliten la incorporación entre ambas comunidades (Kynigos y Kalogeria, 2012). Un ejemplo lo presentan Matranga et al. (2016) al incluir un foro dentro de un programa virtual de formación profesional, en donde a través de la asignación previa de actividades que funcionan como "objetos frontera" generan el intercambio de experiencias entre profesores y educadores matemáticos.

Otro de los puntos de partida al diseñar procesos formativos en línea para profesores de matemáticas es el deseo de favorecer la formación de comunidades de investigación (COI). Este tipo de comunidades, de acuerdo con Garrison et al. (2000), se componen de profesores y estudiantes que se comunican a través de computadoras y logran aprendizajes mediante la interacción de tres elementos centrales: presencia cognitiva, presencia social y presencia del profesor. Cada uno de estos elementos, posee indicadores particulares que sirven como guía a los educadores para mediar los diálogos entre los participantes de forma tal que favorezcan el aprendizaje.

Choppin et al. (2019), describen cómo a través de un proceso de mediación, considerando los principios de las COI, se procura la construcción de significados mediante diversos procesos reflexivos entre profesores de matemáticas participantes en una experiencia de formación continua.

Todas las aproximaciones teóricas mencionadas en los párrafos anteriores se utilizan como puntos de referencia en ciertas partes del diseño instruccional propuesto en espacios de formación en línea para docentes de matemáticas. Sin embargo, se encontró poca información en la literatura sobre experiencias en donde se tomara una perspectiva teórica que pudiera servir no solo como punto de referencia, sino como eje central del diseño de todo un proyecto formativo.

El único modelo de este tipo creado específicamente para el desarrollo profesional de profesores de matemáticas en ambientes virtuales, es el Online Asynchronous Collaboration Model (OAC) planteado por Silverman y Clay (2009b). Sus autores afirman que su construcción se basa en resultados de 
investigación recientes en el área de educación matemática para profesores y que a la par, considera las ventajas de la naturaleza colaborativa del internet y la posibilidad de las interacciones online. Lee et al. (2017), ilustran la implementación de dicha propuesta.

En la revisión de literatura también se encontraron otros modelos de diseño instruccional utilizados en propuestas de formación de profesores de matemáticas, pero se caracterizan por ser adaptaciones a modelos no específicos para educación matemática, por lo que los autores realizan propuestas de adaptación. Por ejemplo, Montiel (2010), cuya propuesta se basa en el diseño de instrucción para ambientes virtuales propuesto por Ally (2004) y Choppin et al. (2019), cuya propuesta formativa es una adaptación del modelo Conjecture Mapping propuesto por Sandoval (2014).

\section{ASPECTOS RELEVANTES QUE NO HAN SIDO SUFICIENTEMENTE INVESTIGADOS}

Es claro que existe un interés dentro de la educación matemática por conocer las innovaciones y beneficios que aportan los entornos virtuales a los procesos de desarrollo profesional docente. De igual forma, existen avances en cuanto a conocer cuáles son los principales referentes teóricos que han sido de utilidad para guiar tanto el diseño como la observación de los procesos que se desarrollan durante este tipo de formación docente. Sin embargo, aún hay varios aspectos relativos a esta área de investigación en los que se cuenta con poca información. En este sentido, Patahuddin (2013) confirma la necesidad de futuras investigaciones que permitan ofrecer una mejor comprensión de cómo el desarrollo profesional a través de entornos virtuales se ajusta a las diversos contextos y necesidades de los profesores, cuáles son los roles que juegan los facilitadores o educadores matemáticos en este tipo de ambientes, qué corrientes pedagógicas son utilizadas y cómo podría aprovecharse la información que proporcionan los macrodatos (big data) generados en estos espacios para una mejor comprensión de la efectividad del desarrollo profesional docente en entornos virtuales.

Particularmente, con relación al rol que juegan los educadores matemáticos en este tipo de formación docente, Bennison et al. (2020), mencionan que se le ha prestado poca atención a conocer bajo qué criterios estos formadores de docentes diseñan y seleccionan los recursos que utilizan durante la 
implementación de estos cursos y cuáles son las estrategias que utilizan para asegurar la calidad de dichos recursos. Asimismo, se conoce poco sobre la forma, frecuencia y profundidad en la que estos educadores matemáticos interactúan con los profesores y los retroalimentan con relación a sus aportaciones.

Desde el punto de vista de los profesores participantes, aún se sabe poco sobre cuáles son los factores que encuentran más relevantes para su formación dentro este tipo de procesos formativos y que favorezcan un impacto positivo dentro de su práctica profesional. Asimismo, existe poca información sobre las emociones y niveles de satisfacción que experimentan durante su participación y la relación que guarda con su desempeño en estos espacios formativos. De igual forma, será necesario identificar cuáles son los factores críticos que favorecen la permanencia de las nuevas comunidades de práctica entre profesores más allá de su participación en algún programa formativo en específico (Borba y Llinares, 2012).

Finalmente, desde el punto de vista de las herramientas tecnológicas utilizadas, si bien, ya existen investigaciones que reportan cómo su utilización impacta tanto en la forma de aprender y aplicar diversos conceptos matemáticos como en la forma en la que se desarrollan los procesos comunicativos. Aún hace falta mayor claridad en cuánto a qué recursos tecnológicos resultan más o menos útiles según los objetivos planteados en cada espacio virtual destinado al desarrollo profesional docente y cómo dichos recursos pueden aprovecharse para ser utilizados como objetos frontera en la formación de comunidades de práctica (Kynigos y Kalogeria, 2012).

En suma, al ser la modalidad virtual, un espacio relativamente reciente utilizado para promover el desarrollo profesional de docentes de matemáticas, existe un gran número de aspectos que aún requieren ser estudiados con más detalle. En este sentido, será necesario revisar continuamente los avances en dicha área de investigación y considerarlos tanto para determinar qué aspectos resultan más relevantes estudiar en un futuro como para conocer qué ventajas ofrecen este tipo de espacios formativos y cuáles son los referentes teóricos que pueden guiar el diseño e implementación de este tipo de espacios. 


\section{REFERENCIAS}

Ally, M. (2004). Foundations of educational theory for online learning. En T. Anderson y F. Elloumi (Eds.). Theory and Practice of Online Learning (pp. 3-31). Athabasca University. Andrade-Molina, M., Montecino, A., y Aguilar, M. (2020). Beyond quality metrics: defying journal rankings as the philosopher's stone of mathematics education research. Educational Studies in Mathematics, 103(3), 359-374.

Bennison, A., Goos, M., Geiger, V. (2020). Utilising a research-informed instructional design approach to develop an online resource to support teacher professional learning on embedding numeracy across the curriculum. ZDM Mathematics Education, 52(5), 1017-1031. Bommel, J., y Liljekvist, Y. (2015). Facebook and mathematics teachers' professional development: Informing our community. En K. Krainer y N. Vondrová (Eds.), Proceedings of the Ninth Congress of the European Society for Research in Mathematics Education (pp. 2930-2936). ERME.

Borba, M. C. (2012). Humans-with-media and continuing education for mathematics teachers in online environments. ZDM Mathematics Education, 44(6), 801-814.

Borba, M. C., y Llinares, S. (2012). Online mathematics teacher education: overview of an emergent field of research. ZDM Mathematics Education, 44(6), 697-704.

Cendros, R., y Gadanidis, G. (2020). Online collaborative mind mapping in a mathematics teacher education program: a study on student interaction and knowledge construction. ZDM Mathematics Education. 52(5), 943-958.

Choppin, J. et al. (2019). Exploring qualities of a community of inquiry in a synchronous online course. En S. Otten, A. G. Candela, Z. de Araujo, C. Haines y C. Munter (Eds.), Proceedings of the forty-first annual meeting of the North American Chapter of the International Group for the Psychology of Mathematics Education (pp.1841-1845). PME.

Clark-Wilson, A., y Hoyles, C. (2019). A research-informed web-based professional development toolkit to support technology-enhanced mathematics teaching at scale. Educational Studies in Mathematics, 102(3), 343-359.

Clay, E., Silverman, J., y Fischer, D. J. (2012). Unpacking online asynchronous collaboration in mathematics teacher education. ZDM Mathematics Education, 44(6), 761-773.

Dean, C., y Silverman, J. (2009). Transferability of an existing framework for analysing teacher's learning in an online professional teaching community. En S. Swars, D. Stinson, y S. Lemons-Smith (Eds.), Proceedings of the 31st annual meeting of the North American Chapter of the International Group for the Psychology of Mathematics Education (pp. 1537-1544). PME.

Erixon, E. (2016). Learning activities and discourses in mathematics teachers' synchronous oral communication online. Research in Mathematics Education, 18(3), 267-282. 
Garrison, D. R., Anderson, T., y Archer, W. (2000). Critical inquiry in a text-based environment: Computer conferencing in higher education. The Internet and Higher Education, 2(2-3), 87-105.

Gueudet, G. et al. (2012). Online paths in mathematics teacher training: new resources and new skills for teacher educators. ZDM Mathematics Education, 44(6), 717-731.

Gueudet, G., y Trouche, L. (2009). Towards new documentation systems for mathematics teachers? Educational Studies in Mathematics, 71(3), 199-218.

Hoyos, V. (2012). Online education for in-service secondary teachers and the incorporation of mathematics technology in the classroom. ZDM Mathematics Education, 44(6), 775-786.

Kynigos, C., y Kalogeria, E. (2012). Boundary crossing through in-service online mathematics teacher education: the case of scenarios and half-baked microworlds. ZDM Mathematics Education, 44(6), 733-745.

Lee, H., Lovett, J., y Mojica, G. (2017). Characterizing impacts of online professional development on teacher's beliefs and perspectives about teaching statistics En E. Galindo y J. Newton (Eds.), Proceedings of the 39th annual meeting of the North American Chapter of the International Group for the Psychology of Mathematics Education (pp. 407-414). Hoosier Association of Mathematics Teacher Educators.

Lezama, J. (2009). Posgrado a distancia en línea en matemática educativa, una alternativa de formación de profesores. La propuesta del Instituto Politécnico Nacional para América Latina. En P. Lestón (Ed.), Acta Latinoamericana de Matemática Educativa (vol. 22, pp. 1585-1595). Colegio Mexicano de Matemática Educativa y Comité Latinoamericano de Matemática Educativa.

Li, Y., y Qi, C. (2011). Online study collaboration to improve teachers' expertise in instructional design in mathematics. ZDM Mathematics Education, 43(6-7), 833-845.

Maass, K. et al. (2019). Different ways to implement innovative teaching approaches at scale. Educational Studies in Mathematics, 102(3), 303-318.

Matranga, A. et al. (2016). Understanding teachers' participation in an emerging online community of practice. En M. B. Wood, E. E. Turner, M. Civil y J. A. Eli (Eds.), Proceedings of the 38th annual meeting of the North American Chapter of the International Group for the Psychology of Mathematics Education (pp. 383-386). PME.

Montiel, G. (2010). Hacia el rediseño del discurso: formación docente en línea centrada en la resignificación de la matemática escolar. Revista Latinoamericana de Investigación en Matemática Educativa, 13(4-I), 69-84.

Müller, D. et al. (2011). Las interacciones en un entorno virtual. Una experiencia en la formación de docentes. En P. Lestón (Ed.), Acta Latinoamericana de Matemática Educativa (vol. 24, pp. 971-980). Colegio Mexicano de Matemática Educativa y Comité Latinoamericano de Matemática Educativa. 
Patahuddin, S. M. (2013). Mathematics teacher professional development in and through internet use: reflections on an ethnographic study. Mathematics Education Research Journal, 25(4), 503-521.

Ponte J. P. et al. (2009) Tools and Settings Supporting Mathematics Teachers' Learning in and from Practice. En R. Even y D. L. Ball (Eds.), The Professional Education and Development of Teachers of Mathematics. The 15th ICMI Study (pp 185-209). Springer.

Sánchez, M. (2010). Internet-based dialogue: A basis for reflection in an in-service mathematics teacher education program. En V. Durand-Guerrier, S. Soury-Lavergne y F. Arzarello (Eds.), Proceedings of the Sixth Congress of the European Society for Research in Mathematics Education (pp. 954-963). ERME.

Sandoval, W. (2014). Conjecture mapping: An approach to systematic educational design research. Journal of the Learning Sciences, 23(1), 18-36.

Shulman, L. S. (1986). Those who understand: Knowledge growth in teaching. Educational Researcher, 15(2), 4-14.

Silverman, J., y Clay, E. (2009a). Supporting teachers' development of coherence through a focus on mathematical structure. En S. L. Swars, D.W. Stinson, y S. Lemons-Smith (Eds.), Proceedings of the 31st annual meeting of the North American Chapter of the International Group for the Psychology of Mathematics Education (pp. 1352-1359). PME.

Silverman, J., y Clay, E. L. (2009b). Online asynchronous collaboration in mathematics teacher education and the development of mathematical knowledge for teaching. The Teacher Educator, 45(1), 54-73.

Surrette, T. N., y Johnson, C. C. (2015). Assessing the ability of an online environment to facilitate the critical features of teacher professional development. School Science and Mathematics, 115(6), 260-270.

Thomas, M. O. J., y Hong, Y. Y. (2013). Teacher integration of technology into mathematics learning. International Journal of Technology in Mathematics Education, 20(2), 69-84.

Toerner, G., y Arzarello, F. (2012). Grading mathematics education research journals. Newsletter of the European Mathematical Society, 86, 52-54.

Wenger, E. (2001). Comunidades de práctica. Aprendizaje, significado e identidad. Paidós. Williams, S., y Leatham, K. (2017). Journal quality in mathematics education. Journal for Research in Mathematics Education, 48(4), 369-396.

FÁtima WidMAn Aguayo

Dirección: Calz. Legaria 694, Col. Irrigación, CP 11500, Miguel Hidalgo, Ciudad de México

Teléfono: (55) 57296000 